\title{
BMJ Open Effect of a national requirement to introduce named accountable general practitioners for patients aged 75 or older in England: regression discontinuity analysis of general practice utilisation and continuity of care
}

\author{
Isaac Barker, Therese Lloyd, Adam Steventon
}

To cite: Barker I, Lloyd T, Steventon A. Effect of a national requirement to introduce named accountable general practitioners for patients aged 75 or older in England: regression discontinuity analysis of general practice utilisation and continuity of care. BMJ Open 2016;6:e011422. doi:10.1136/bmjopen-2016011422

- Prepublication history and additional material is available. To view please visit the journal (http://dx.doi.org/ 10.1136/bmjopen-2016011422).

Received 5 February 2016 Revised 8 June 2016 Accepted 9 June 2016

CrossMark

Data Analytics, The Health Foundation, London, UK

Correspondence to Isaac Barker; isaac.barker@health.org.uk

\section{ABSTRACT}

Objective: To assess the effect of introducing named accountable general practitioners (GPs) for patients aged 75 years on patterns of general practice utilisation, including continuity of care.

Design: Regression discontinuity design applied to data from the Clinical Practice Research Datalink to estimate the treatment effect for compliers aged 75.

Setting: 200 general practices in England.

Participants: 255469 patients aged between 65 and 85, after excluding those aged 75.

Intervention: From April 2014, general practices in England were required to offer patients aged 75 or over a named accountable GP. This study compared having named accountable GPs for patients aged just over 75 with usual care provided for patients just under 75 .

Outcomes: Number of contacts (face-to-face or telephone) with GPs, longitudinal continuity of care (usual provider of care, or UPC, index), number of referrals to specialist care and numbers of common diagnostic tests. Outcomes were measured over 9 months following assignment to a named accountable GP and for a comparable period for those unassigned.

Results: The proportion of patients with a named accountable GP increased from $3.5 \%$ to $79.8 \%$ at age 75. No statistically significant effects were detected for continuity of care (estimated treatment effect 0.00 , $95 \% \mathrm{Cl}-0.01$ to 0.02 ) or the number of GP contacts per person (estimated treatment effect $-0.11,95 \% \mathrm{Cl}$ -0.31 to 0.09 ) over 9 months. No significant change was seen in the number of referrals, blood pressure or $\mathrm{HbA1c}$ diagnostic tests per person. A statistically significant treatment effect of -0.05 cholesterol tests per person $(95 \% \mathrm{Cl}-0.07$ to -0.02$)$ was estimated; however, sensitivity analysis indicated that this effect predated the introduction of named accountable GPs. Conclusions: Continuity of care is valued by patients, but the named accountable GP initiative did not improve continuity of care or change patterns of GP utilisation in the first 9 months of the policy.

\section{Strengths and limitations of this study}

- The paper provides an evaluation of a national policy that aimed to improve continuity of care for elderly patients.

- The nature of the decision rule used to determine eligibility for this treatment meant that it was appropriate to employ a regression discontinuity design. This design is an effective way to deal with confounding, a common problem in many observational studies.

- The study used a large, patient-level data set from the Clinical Practice Research Datalink which meant that we had statistical power to detect small treatment effects.

- The study period (9 months) might be too short to realise the full benefits of the policy.

- The estimated treatment effects are for compliers at age 75 so might not be generalisable to other ages.

\section{INTRODUCTION}

Continuity of care has historically been a strength of general practice in England, in part due to its gatekeeping role for specialist care and the long-standing availability of electronic medical records. It is highly valued by patients and especially by older people. ${ }^{1}$ However, general practice has changed rapidly over the last decade, with the end to personal registration with a general practitioner (GP), increasing specialisation of primary care, organisational separation of daytime and out-of-hours services, an increase in federated practices and a focus on improving rapid access to primary care. There are signs that continuity is falling - the proportion of people reporting they could see their preferred GP 'always or 
almost always' fell slightly from $40.8 \%$ to $35.3 \%$ between 2012 and 2016. ${ }^{1}$ Addressing continuity of care was identified as a key priority in the English NHS Primary Care plan laid out in 'Transforming Primary Care', and in 2014 resources were set aside for this purpose. ${ }^{2}$

The pay-for-performance scheme for general practice (the quality and outcomes framework, or QOF) was reformed in 2014, and 341 of 900 points were removed (equating to $£ 416$ million of funding). Alongside new financial incentives to promote proactive care management for at-risk patients (the Avoiding Unplanned Admissions Enhanced Service (AUAES)), general practices saw a substantial increase in core funding from $£ 66$ to $£ 74$ per patient per year in April 2014 (around £290 million). ${ }^{3}$ At the same time, a requirement was added to the contract, stipulating that general practices were expected to offer all patients aged 75 years or over a named accountable GP to take lead responsibility for ensuring the delivery of relevant health and social care. ${ }^{4}$ The requirement to offer named accountable GPs was broadened to cover patients of all ages from April 2015 and has remained in the national contract for 2016.

The importance of improving continuity of care is evident from at least 19 research studies (including 4 clinical trials) indicating its association with patient satisfaction. ${ }^{5}$ The relationship between continuity and patient outcomes has been less clear, partly because continuity is a complex, multifaceted concept reflecting at least three domains: ${ }^{5}$ the subjective experience of a caring relationship ('interpersonal continuity'); a history of interacting with the same healthcare professional across a series of discrete episodes ('longitudinal continuity') and the availability of clinical and psychosocial information across encounters and professionals ('informational continuity'). Some of these domains have been linked to utilisation for example, the ability of a patient to see their preferred GP has been associated with lower unplanned hospital admissions at general practice level, ${ }^{6}$ and a sustained increase in interpersonal continuity may lead to reduced referrals to specialist care. ${ }^{7}$ However, few intervention studies have satisfactorily demonstrated improved health outcomes as a result of initiatives to improve the continuity of care. A systematic review and meta-analysis of case-management policies found that 9 of the 11 identified trials showed no significant reductions in unplanned hospital admissions when compared with usual care. ${ }^{8}$ This is in part because non-randomised studies may not deal adequately with selection bias.

In this paper, we use national data to assess whether the introduction of named accountable GPs was associated with changes in primary care utilisation and longitudinal continuity of care at age 75 . The study will provide a benchmark for the quality improvement activities announced in the 2016-2017 contract for general practice care-previous studies have illustrated that, without sustained attention to implementation, new approaches to delivering care for complex patients often fail to improve continuity and thus prevent undesirable and costly events, such as unplanned hospital admissions. ${ }^{8}{ }^{10}$ The initial version of the named accountable GP policy evaluated here required a prescribed set of administrative activities (eg, recording in the electronic medical records that patients were informed of their named accountable GP within 21 days of their 75 th birthday) ${ }^{4}$ without detailed guidance about how workflows could be adapted to improve continuity (eg, through changes to appointment booking systems). ${ }^{11}$ However, it is possible that, by informing service users of their entitlement for named GPs, these groups might be more empowered to request appointments with their preferred doctor.

By applying a regression discontinuity design, we avoid the problem with selection bias present in many nonrandomised studies and thus add to the literature on the effect of these interventions to improve continuity and patient outcomes. Our study end points include a measure of continuity of care, the number of contacts with GPs, the frequency of common diagnostic tests and numbers of referrals to specialist care. Greater continuity of care could affect these other metrics in either direction, since greater continuity might lead either to a rationalisation of care or to the identification of unmet need. ${ }^{7}$

\section{METHODS}

The design of the general medical services contract offers an opportunity to apply a regression discontinuity design, thereby avoiding confounding due to observed and unobserved characteristics, a common concern in observational studies. ${ }^{12}$ Regression discontinuity designs are appropriate in situations such as this where eligibility for an intervention changes sharply at a predefined threshold (here, age 75); this is a common situation in healthcare, but regression discontinuity designs are underused. ${ }^{13} \mathrm{We}$ show that it is reasonable to assume that patients aged just below and just above 75 are similar at baseline in terms of all measured and unmeasured characteristics. Therefore, we can assign any change in their outcomes at age 75 to a change in management at this age. Regression discontinuity designs have been shown to replicate the results of randomised control trials. ${ }^{14} 15$

The study was conducted in line with the original study protocol, ${ }^{16}$ except that in this paper we assessed end points over 9 rather than 6 months. We did this because, in our data, patients experienced fewer contacts than anticipated, and a longer study period allowed us to measure continuity of care more precisely. Six-month end points showed similar patterns to those reported here. The study protocol was reviewed by the Independent Scientific Advisory Committee for the Clinical Practice Research Datalink (CPRD).

\section{Intervention}

Under the 2014-2015 National Health Service contract for general medical services in England, all general practices were obliged to offer a named accountable GP to 
patients who were permanently registered with the practice and aged 75 or over. This GP would "take lead responsibility for ensuring that all appropriate services required under the contract are delivered", ${ }^{4}$ including health and social care. A particular requirement related to the intervention was the delivery of the over 75 health check, an annual health assessment that has been in place for this age group since 2004.

General practices could inform the patient of their named accountable GP by any means, though they were provided with a template letter (http://www.nhsemployers. org/your-workforce/primary-care-contacts/general-medicalservices/gms-contract-changes/contract-changes-2014-15) that outlined to patients the role of this clinician in their care. The template informed patients of which GP had been assigned to them and stated that no action was necessary on the patient's part, though general practices were obliged to make a reasonable effort to accommodate patients' choice of GP. Patients had no obligation to see their named accountable GP, and the policy neither explicitly encouraged nor physically aided changes to appointment booking systems.

Practices had until 30 June 2014 to notify patients who were already aged 75 or over at the beginning of the financial year. Thereafter, patients were to be notified of their named accountable GP within 21 days of their 75th birthday. The general medical services contract introduced a Read code for 'patient notified of named general practitioner'; this Read code practices were required to use to record on electronic medical records that they had assigned a patient a named accountable GP so that NHS England area teams could check practices met their contractual requirements. The Read code was not used to determine eligibility for payment. ${ }^{17}$ We compared assignment of a named accountable GP for patients aged 75 with usual care for patients aged just under 75 , who at the time of our study were not entitled to named accountable GPs.

\section{Data sets}

We obtained general practice electronic medical records for patients aged between 65 and 85 from CPRD. ${ }^{18}$ This included data on contacts with healthcare professionals, details of diagnostic tests, referrals to specialist healthcare, diagnosed health conditions, demographic characteristics and the start and end dates of patient registrations. The CPRD supplied linked Hospital Episode Statistics data (to March 2014); information on registered deaths (to April 2014); and patient-level socioeconomic deprivation scores (Index of Multiple Deprivation, measured at small area level in 2010). ${ }^{19}$ All data were pseudonymised before transfer to the research team, and in particular the date of birth was replaced with year of birth. ${ }^{20}$

\section{Study cohort}

We received records for all English general practices submitting data to CPRD that were eligible for linkage to other data sources, except for practices whose data had been identified by CPRD as not being up to research standard, and practices that at the time had not submitted data for the entirety of the 2014-2015 financial year.

Study participants were registered patients born between 1929 and 1949 and so were aged between 65 and 85 in 2014 . We excluded patients identified by practices as being at particular risk of unplanned hospital admission and entered on a case-management register for the purposes of the AUAES, since those patients were offered a named accountable GP irrespective of their age. We also excluded patients who deregistered from the general practice during the 9-month follow-up period. Finally, although patients born in 1939 reached their 75 th birthdays in 2014, we were not able to identify precisely when this happened, due to the lack of information on their full date of birth. These patients were therefore omitted from the analysis, but, as a result, our modelling required some extrapolation (see below).

We identified patients who received a named accountable GP between 1 April 2014 and 30 June 2014, using the Read code that was specified as part the general medical services contract. Patients receiving the intervention within this period were attributed a set of baseline variables and study end points based on the date on which the patient was recorded as having received a named accountable GP. For other patients, 'index dates' were randomly assigned such that the distribution of index dates across months was the same as for the patients assigned a named accountable GP. Baseline variables and study end points were then calculated using these index dates.

\section{Study end points}

We assessed longitudinal continuity of care using the usual provider of care (UPC) index, ${ }^{21}{ }^{22}$ a widely used metric that is defined as the proportion of a patient's general practice contacts that were with the most frequently seen GP. ${ }^{22}{ }^{23}$ For example, if, over a certain period, a patient had five contacts including three with the same GP, then the usual provider of care index would be 0.6 . We calculated the usual provider of care index with reference to the most frequently seen GP, rather than the named accountable GP, since the latter was not identifiable from the patient records and in any case was not applicable for study participants who did not receive a named accountable GP. ${ }^{24}$

The theory behind the intervention is such that, in the absence of improvements to continuity of care, it seemed unlikely that we would find changes to the number of GP contacts, referrals to specialist care or numbers of diagnostic tests. However, in the study protocol, we specified that we would analyse these end points regardless of the findings for the usual provider of care index, since it is theoretically possible that, for example, the letters led to an increase in enquiries from patients without improvements to continuity. We identified GP contacts from the data by selecting records corresponding to relevant types 
of encounter (eg, clinic, home visit and telephone) entered by a GP (eg, partner, registrar, sole practitioner or locum GP). Diagnostic tests were limited to blood pressure tests, cholesterol tests and, for patients with diabetes, haemoglobin 1Ac tests. Referrals to specialist care were defined as any referrals, including self-referrals, to any type of specialist healthcare practitioner, including specialist nursing. Number of tests and referrals were limited to one per patient per day.

We measured study end points over 9 months following the index dates, so that no patients were followed up beyond April 2015 when named accountable GPs were rolled out to adults of all ages. Our sample size was greater than the number of observations needed to estimate a statistically significant change of $5 \%$ in the number of GP contacts relative to usual care, based on an initial calculation. ${ }^{16}$ The usual provider of care index was not chosen as the primary study end point since it was defined only for the subgroup of patients who experienced at least two GP contacts during the follow-up period, and it was an intermediate outcome since the ultimate aim of the policy was to improve patient outcomes.

\section{Statistical methods}

We anticipated that some patients would be assigned a named accountable GP even though they were aged below 75 years, whereas some patients older than age 75 would not be offered a named accountable GP. Therefore, we employed a fuzzy regression discontinuity design to estimate the effect of being assigned a named accountable GP for compliers (ie, for those patients who would in practice receive a named accountable GP if eligible under the policy, but not receive one if ineligible). ${ }^{25}$ As sensitivity analysis, we employed a sharp regression discontinuity design to estimate an average treatment effect across all people aged 75, like intention-to-treat analysis of randomised data.

The regression models used to estimate these treatment effects were similar to those from a previous paper and were applied to patients within a certain number of years of age 75 (ie, aged within a certain 'bandwidth'). ${ }^{26}$ The models allowed for a difference in the level and slope of study end points at age 75 and likewise for a change in the level and slope of the percentage of patients at each age receiving a named accountable GP (see technical appendix, online supplementary material). As noted above, we conducted a limited amount of extrapolation when estimating treatment effects, since the lack of full date of birth meant that we excluded patients born in 1939. Thus, for example, adopting a bandwidth of 4 years meant that we estimated outcomes under the control treatment by fitting a model on data for people aged between 70 and 74 and then extrapolating this model to estimate outcomes under control at age 75 . We tested several bandwidths and selected the order of the polynomial function used in the regression models using a combination of formal goodness of fit tests, visual analysis of the data and previous literature. ${ }^{27}$ We assessed the plausibility of our model estimates graphically by examining our outcomes binned by the assignment variable. A bias-correction method was necessary because the lack of full date of birth meant that age could only be approximated. ${ }^{26}$ Bootstrapped 95\% CIs are reported for the estimated treatment effects for each of the study end points. ${ }^{28}$

\section{Design validity}

The regression discontinuity design makes a limited number of assumptions that were plausible in our setting; the main assumption is that individuals do not have precise control over the variable that is used to determine treatment eligibility (ie, recorded age). However, we validated the underlying assumptions graphically according to recommended practice. ${ }^{25}$ First, we checked that there was a large increase in the percentage of patients assigned to receive a named accountable GP at age 75. Second, we confirmed that there was no sudden change in the number of registered patients at age 75 , since such a discontinuity might imply that patients had control over their recorded age. Finally, we checked that there were no discontinuities at age 75 in the following baseline variables: gender; ethnicity; region; socioeconomic deprivation score; number of chronic health conditions; ${ }^{29}$ number of hospitalisations ending within 9 months prior to the index dates; and the equivalents of the study end points, but calculated over the 9 months prior to the index date.

\section{Sensitivity analyses}

We tested the sensitivity of our estimates to model specification using higher and lower order polynomials and narrower bandwidths. We also tested sensitivity to the way that our primary end points were coded by (1) including contacts with practice nurses; (2) restricting the analysis to face-to-face GP contacts and (3) restricting the analysis of the usual provider of care index to patients with a minimum of three contacts rather than two contacts during the study period. Finally, we were aware that, should we find a treatment effect at age 75 , this might have been attributable to the over-75 health check, which predated the named accountable GP policy. Therefore, we compared treatment effects in the 2014-2015 study period with those estimated using similar data but from 1 year earlier, using a sharp design in both cases since the named accountable GP did not exist in the earlier time period.

\section{RESULTS}

After applying the inclusion and exclusion criteria, our data set comprised 267804 patients from 200 general practices (figure 1), of which 12335 patients aged 75 were removed, leaving 167213 patients aged under 75 years and 88256 patients aged above 75 years. The percentage of patients assigned a named accountable 
Figure 1 Diagram showing the flow of patients into the study. AUAES, Avoiding Unplanned Admissions Enhanced Service; CPRD, Clinical Practice Research Datalink.

\section{Patient definition}

From the source population in CPRD GOLD ( $N=13811$ 287):

- 1963186 patients (686 practices) born between 1929 and 1949 (inclusive)

- 1963186 patients (686 practices) meet gender criterion (Male, Female and Indeterminate Only)

- 664321 patients (476 practices) are alive and are registered with an up-to-standard GP practice on the $1^{\text {st }}$ April 2014

- 484718 patients (348 practices) registered at a GP practice still contributing data on the $31^{\text {st }}$ March 2015 (i.e. last collection date for practice is on or after $1^{\text {st }}$ April 2015)

- 294562 patients (200 practices) registered at an English GP practice and eligible for linkage - 294562 patients received from CPRD

- 5 patients contained no date of birth, one was flagged as 'unacceptable', and 44 were not alive after 01/04/2014

294412 patients 'acceptable' and alive after 01/04/2014

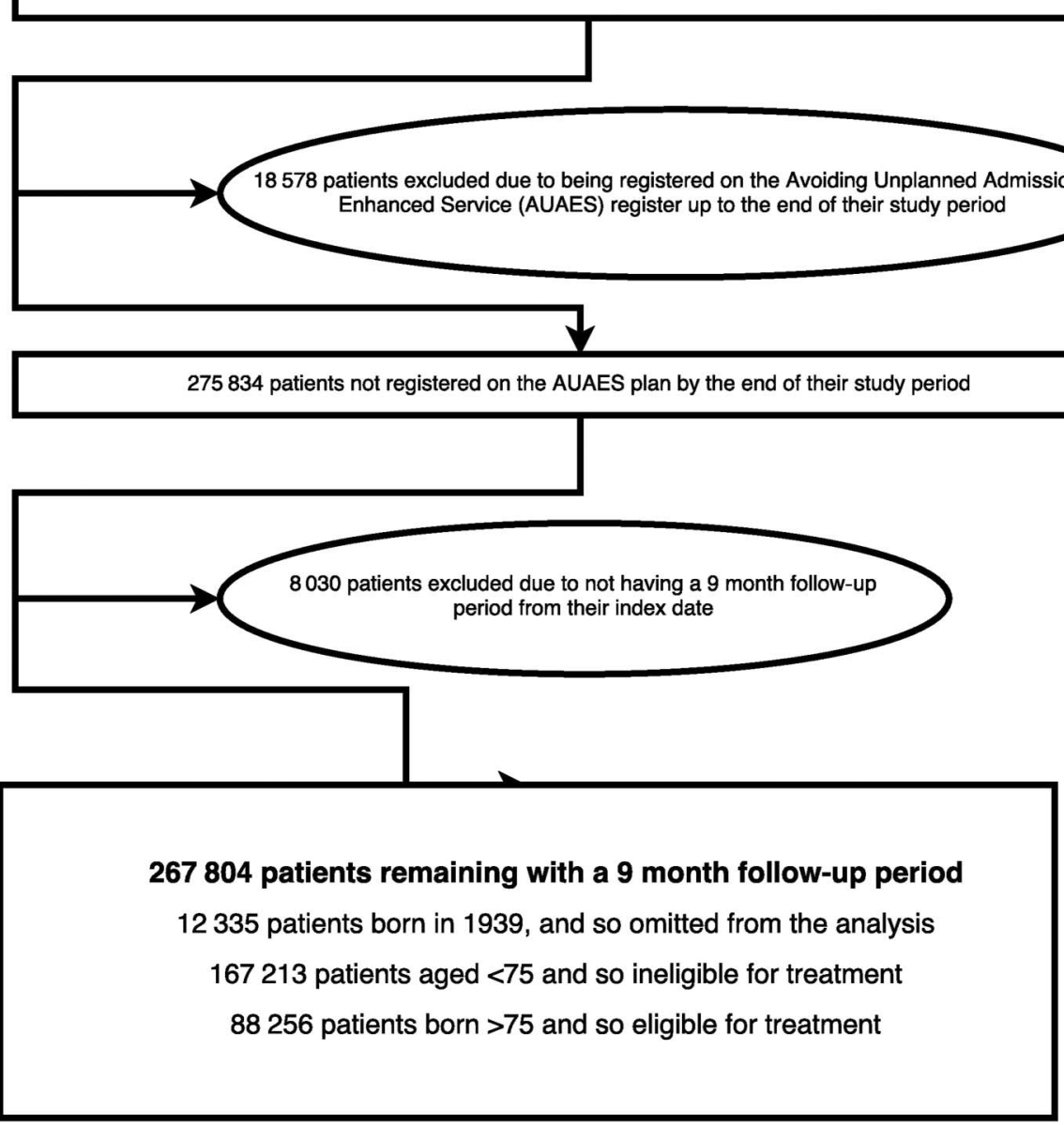

GP increased from $3.5 \%$ just under age 75 to $79.8 \%$ just over 75 - an increase of $76.2 \%$ (figure 2). No discontinuities were found at age 75 in any of the baseline variables, and likewise the number of registered patients did not change at age 75 (see online supplementary materials 1 and 2).

Assignment to a named accountable GP was associated with 0.11 fewer GP contacts per patient over 9 months at age 75 , but this did not reach statistical significance (95\% CI -0.31 to $0.09, \mathrm{p}=0.27$, see table 1 and figure 3 , "Number of GP contacts"). A change of 0.11 GP contacts per person is equivalent to a relative change in contacts of around $-2.58 \%$ (95\% CI $-7.13 \%$ to $1.97 \%)$. The usual provider of care index did not show a trend with age, and no discontinuity was visible at age 75 (estimated treatment effect $0.00,95 \%$ CI -0.01 to 0.02 , $\mathrm{p}=0.467$, see table 1 and figure 3, "Usual provider of care index"). Assignment to a named accountable GP was associated with fewer cholesterol tests per person $(-0.05,95 \%$ CI -0.07 to $-0.02, \mathrm{p}<0.001)$, but no statistically significant differences were found for numbers of referrals to specialist care, blood pressure tests and HbAlc tests among patients with diabetes (table 1 and figure 3 ).

\section{Sensitivity analysis}

Our findings were not sensitive to whether we restricted our analysis to face-to-face interactions. Likewise, findings for the usual provider of care index did not change when we restricted the analysis to patients with a minimum of three, rather than two, contacts over the 9 


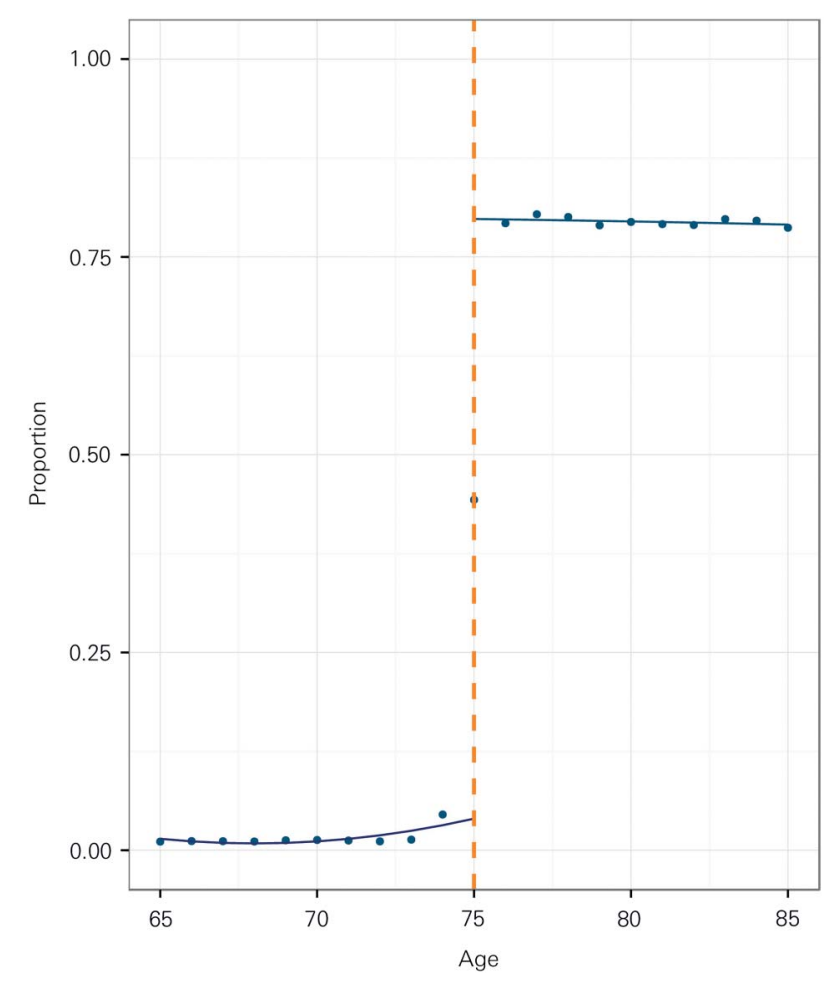

Figure 2 Proportion of patients assigned a named accountable general practitioner (GP), by year $(n=255469)$.

month study period (see online supplementary material 4). Although results for some other outcomes achieved statistical significance in sensitivity analysis, no outcome demonstrated clinically meaningful impacts or consistent results across alternative polynomial orders and bandwidths (see online supplementary materials 4,5 and 6 ). Analysis of 2013-2014 data revealed that there was a statistically significant decline in number of cholesterol tests at age 75 also in 2013-2014, before the policy was implemented $(-0.03,95 \%$ CI -0.05 to -0.00$)$ (see online supplementary material 7).

\section{DISCUSSION}

Within 3 months of the new contract being introduced in April 2014, almost $80 \%$ of patients aged between 75 and 85 had been assigned a named accountable GP. If extrapolated nationally to all patients aged over 75 , this means that around 3.6 million patients in England were informed of their named accountable GP during this period. Despite the administrative effort involved, patients aged just above 75 and receiving a named accountable GP did not experience marked differences in numbers of GP contacts and continuity of care compared with patients receiving usual care and aged just below 75. Likewise, named accountable GPs did not lead to clinically significant changes in referrals to specialist care and common diagnostic tests. These results constitute the first evaluation of the named accountable GP policy, which has since been expanded to include patients of all ages in England, and provide a benchmark for the quality improvement efforts planned in this area.

\section{Strengths and limitations}

Observational studies such as this one are valuable for examining the effectiveness of changes to healthcare in routine settings. However, a common concern is that, in the absence of randomisation, treated and untreated groups might differ at baseline in terms of characteristics that might be observed or unobserved. ${ }^{12}$ To address potential selection bias, many observational studies make the strong and sometimes implausible assumption that all confounders of treatment selection have been observed. However, in this case the nature of the eligibility rules allowed us to employ a regression discontinuity method and to make weaker (hence more plausible) assumptions, namely, that the decision about whether or not to assign a patient a named accountable GP was influenced by whether or not the patient was aged over 75 years, and also that age could not be precisely manipulated in the data. These assumptions are plausible and supported by our identification tests (see online supplementary materials 1 and 2).

Some limited extrapolation was needed when estimating treatment effects, since the lack of information on full date of birth meant that the age of patients could only be measured to a whole year and furthermore, we had to remove individuals born in 1939 from our data

Table 1 Estimated treatment effect for compliers aged 75 years over 9 months from the index date $(n=255469)$

\begin{tabular}{|c|c|c|c|c|c|}
\hline & $\begin{array}{l}\text { Absolute } \\
\text { treatment } \\
\text { effect }\end{array}$ & $\begin{array}{l}\text { Absolute } \\
95 \% \mathrm{Cl}\end{array}$ & $\begin{array}{l}\text { Relative } \\
\text { treatment } \\
\text { effect }(\%)\end{array}$ & $\begin{array}{l}\text { Relative } \\
95 \% \text { Cl }\end{array}$ & p Value \\
\hline Number of GP contacts per patient & -0.111 & -0.307 to 0.085 & -2.58 & $-7.13 \%$ to $1.97 \%$ & 0.267 \\
\hline Usual provider of care index $\dagger$ & 0.004 & -0.007 to 0.016 & 0.66 & $-1.12 \%$ to $2.43 \%$ & 0.467 \\
\hline Number of blood pressure tests per patient & -0.056 & -0.117 to 0.004 & -4.91 & $-10.20 \%$ to $0.38 \%$ & 0.069 \\
\hline Number of cholesterol tests per patient & $-0.047^{\star}$ & -0.073 to -0.021 & -9.11 & $-14.13 \%$ to $-4.08 \%$ & $<0.001$ \\
\hline Number of $\mathrm{HbA} 1 \mathrm{c}$ tests per patientł & 0.012 & -0.130 to 0.153 & 0.67 & $-7.60 \%$ to $8.95 \%$ & 0.873 \\
\hline Number of referrals to specialist care per patient & 0.015 & -0.021 to 0.050 & 3.35 & $-4.90 \%$ to $11.59 \%$ & 0.426 \\
\hline
\end{tabular}


Figure 3 Study end points by year of age. GP, general practitioner. ( $n=$ refers to number of patients).
Number of GP contacts

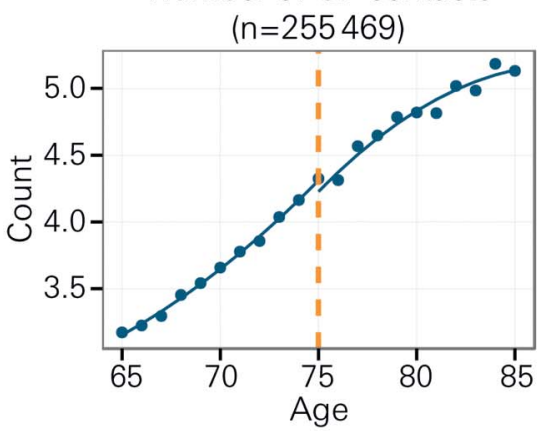

Number of blood pressure tests $(n=255469)$

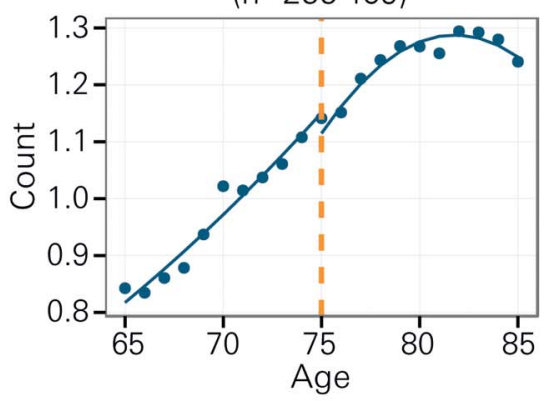

Number of $\mathrm{HbA} 1 \mathrm{c}$ tests

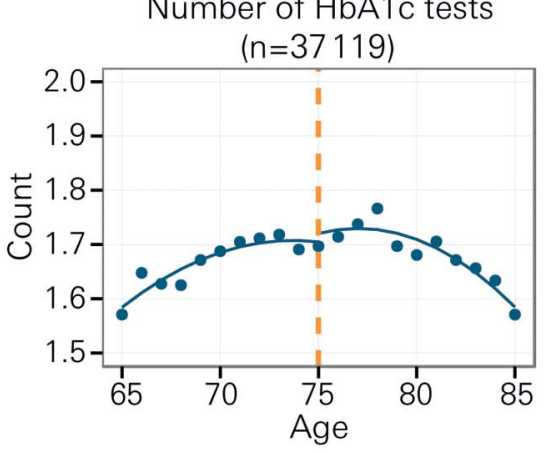

Usual provider of care index $(n=168956)$

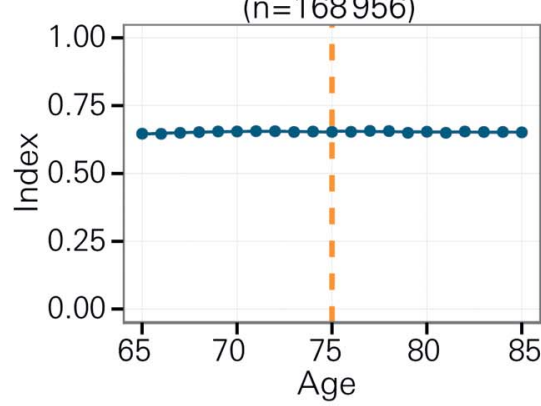

Number of cholesterol tests $(n=255469)$

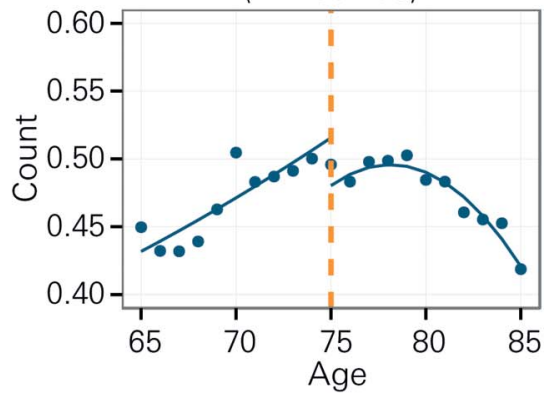

Number of referrals to specialist care $(n=255469)$

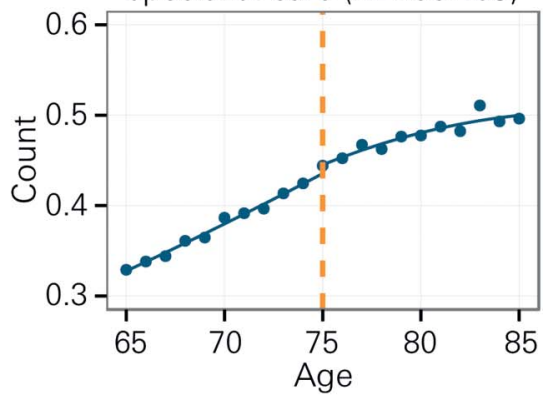

set. However, sensitivity analysis (see online supplementary materials 5 and 6) showed that our findings were robust to model specification. In any case, no clear discontinuity was apparent at age 75 in any of the end points (figure 3). A possible exception was the number of cholesterol tests, but as a similarly sized reduction in cholesterol tests occurred at age 75 in the year preceding the introduction of named accountable GPs, this finding cannot be attributed to named accountable GPs. The absence of statistically significant effects is unlikely to be due to limited sample size, since the study was powered to detect a $5 \%$ relative change in the number of GP contacts, much smaller than other studies. ${ }^{30}$

Although the general practices participating in the CPRD were broadly representative of England, ${ }^{18}$ our estimated treatment effects apply only to compliers aged 75 who were not identified as being in the $2 \%$ highest risk group for unplanned admissions. ${ }^{31}$ Our analysis of longitudinal continuity was based on a subset of patients who had at least two contacts with a GP over the study period. While this meant that our findings for this end point related to only two-thirds of people aged 75 in these practices, longitudinal continuity of care may be less relevant to people who use services less frequently. We could not rule out the possibility that the introduction of named accountable GPs was beneficial to groups of patients not analysed in this study, including those aged younger or older than 75 years. ${ }^{32}$ Although the policy was rolled out to all ages in 2015-2016, practices were no longer required to contact each patient to inform them of their named accountable GP.

A limitation of the data meant that the analysis was blind to whether consultations were actually with the patients' named GP, and instead the most regularly seen GP was used to calculate an index of continuity. Our chosen metric of continuity of care (the usual provider of care index) had an upper bound of 1 and $15 \%$ of patients reached this maximum (see online supplementary material 3); therefore, the estimated treatment effectiveness for this end point might have been dampened by a ceiling effect. However, we used an established measure of continuity and our approach enabled the study end points to be defined comparably between study groups. 
We considered a range of metrics relating to patterns of general practice utilisation, but we could not assess the quality of the interactions from the patient perspective, patient satisfaction with services or interpersonal continuity of care. We did not assess emergency department attendances and hospitalisations since changes to these end points could not plausibly be attributed to the intervention if they were not preceded by changes to patterns of general practice utilisation. Information on community healthcare, social care and access and waiting times in primary care were not available. We did not assess the economic costs. Finally, the benefits of named accountable GPs might only be realised over periods of time longer than those considered in this study. Since named accountable GPs were introduced to adults of all ages from April 2015, we were not able to study periods longer than 9 months.

\section{Mechanisms and interpretation}

Our analysis confirms that general practices in general met their contractual requirement to inform patients of their named accountable GP, but with no concomitant improvement in measured outcomes over 9 months. Of particular concern given the aims of the policy is the lack of effect on the usual provider of care index. This suggests that either the context was not conducive to the proposed changes, or the changes made were not sufficient to improve the continuity of care. Ferris and colleagues $^{33}$ outlined a number of elements necessary to improve longitudinal continuity and coordination of care: approaches must be targeted at patients at-risk of adverse health outcomes, must establish healthcare needs and then effectively address them. The named accountable GP policy was broadly targeted at all patients aged 75 or over and did not include guidance or support about how services could be adapted within a challenging context. GPs in the UK have significantly less time per patient and are more dissatisfied about this than international comparators. ${ }^{34} 35$ However, medical students pursue a career in general practice due to their desire to provide longitudinal care for patients ${ }^{36}$ and to have close involvement in the psychosocial aspects of medical care. ${ }^{37}$

Further analysis could explore whether the effect of named accountable GPs varied between general practices. ${ }^{38}$ For instance, there is strong evidence that unplanned admission rates are significantly correlated with rates of socioeconomic deprivation, ${ }^{39}$ and so an effort to improve continuity may be more effective in practices in more deprived localities. ${ }^{6}$ Achieving better continuity of care may also be predicated on patients requesting appointments specifically with their named accountable GP. Future work could explore the barriers faced from the patient perspective to achieve continuity of care.

\section{CONCLUSIONS}

The introduction of named accountable GPs in England did not improve longitudinal continuity of care over
9 months. Consequently, no changes were detected in numbers of general practice visits, referrals to specialist care and diagnostic tests. Continuity of care is valued by patients and may also be associated with better patient outcomes, so efforts to improve this dimension of quality seem warranted. However, initiatives may be more effective if they more comprehensively address the context in which general practice operates and include support to change workflows.

Contributors AS and TL developed the concept and study design. TL acquired and prepared the data. IB performed the analysis and prepared the draft manuscript. All the authors reviewed and edited the manuscript. AS is the guarantor.

Funding This research received no specific grant from any funding agency in the public, commercial or not-for-profit sectors.

\section{Competing interests None declared.}

Ethics approval The protocol was submitted to and approved by the ISAC committee.

Provenance and peer review Not commissioned; externally peer reviewed.

Data sharing statement No additional data available, due to the CPRD contact signed for this study. Technical appendix included in the online supplementary material.

Open Access This is an Open Access article distributed in accordance with the Creative Commons Attribution Non Commercial (CC BY-NC 4.0) license, which permits others to distribute, remix, adapt, build upon this work noncommercially, and license their derivative works on different terms, provided the original work is properly cited and the use is non-commercial. See: http:// creativecommons.org/licenses/by-nc/4.0/

\section{REFERENCES}

1. Ipsos MORI. GP Patient Survey-National summary report: January 2015 publication. 2015. https://gp-patient.co.uk/surveys-and-reports

2. Department of Health. Transforming primary care. London: Department of Health, 2014. https://www.gov.uk/government/ uploads/system/uploads/attachment_data/file/304139/Transforming_ primary_care.pdf (accessed 10 May 2015).

3. NHS England. Implementing the 2014/15 GP contract. 2014. https:// www.england.nhs.uk/wp-content/uploads/2014/03/gp-cont-changefin.pdf

4. NHS England. General Medical Services contract 2014/15Guidance and audit requirements. NHS Employers, 2014.

5. Saultz JW, Albedaiwi W. Interpersonal continuity of care and patient satisfaction: a critical review. Ann Fam Med 2004;2:445-51.

6. Bankart MJ, Baker R, Rashid A, et al. Characteristics of general practices associated with emergency admission rates to hospital: a cross-sectional study. Emerg Med J 2011;28:558-63.

7. Little P, Everitt $\mathrm{H}$, Williamson $\mathrm{I}$, et al. Observational study of effect of patient centredness and positive approach on outcomes of general practice consultations. BMJ 2001;323:908-11.

8. Huntley AL, Thomas R, Mann M, et al. Is case management effective in reducing the risk of unplanned hospital admissions for older people? A systematic review and meta-analysis. Fam Pract 2013;30:266-75.

9. NHS England. NHS England Letter: GMS Contract Changes 2016/ 17. 2016;1. https://www.england.nhs.uk/commissioning/gp-contract/

10. Gravelle H, Dusheiko M, Sheaff R, et al. Impact of case management (Evercare) on frail elderly patients: controlled before and after analysis of quantitative outcome data. BMJ 2007;334:31.

11. Roland M. Continuity of care: betrayed values or misplaced nostalgia. Int J Integr Care 2012;12:e200.

12. Rubin DB. On the limitations of comparative effectiveness research. Stat Med 2010;29:1991-5.

13. Venkataramani AS, Bor J, Jena AB. Regression discontinuity designs in healthcare research. BMJ 2016;352:i1216.

14. Lee DS, Card D. Regression discontinuity inference with specification error. J Econom 2008;142:655-74.

15. Lee DS, Lemieux T. Regression discontinuity designs in economics. Journal of Economic Literature 2010 Jun;48:281-355. 
16. Lloyd T, Steventon A. Effect of named, accountable GPs on continuity of care: protocol for a regression discontinuity study of a national policy change. Int J Integr Care 2016;16:6.

17. Health and Social Care Information Centre. New GMS Contract Implementation, Dataset and Business Rules-Over 75s Accountable GP. Published Online First: 2014. http://digital.nhs.uk/ media/15754/Over-75s-Accountable-GP-ESv40/pdf/Over_75s_ Accountable_GP_v4.0.pdf

18. Herrett E, Gallagher AM, Bhaskaran K, et al. Data resource profile: Clinical Practice Research Datalink (CPRD). Int J Epidemiol 2015;44:827-36.

19. Office for National Statistics. Lower Layer Super Output Area (LSOA) Boundaries. 09/02/2010. 2010. http://data.gov.uk/dataset/ lower_layer_super_output_area_Isoa_boundaries

20. Boyd P. The requirements of the Data Protection Act 1998 for the processing of medical data. J Med Ethics 2003;29:34-5.

21. Dreiher J, Comaneshter DS, Rosenbluth $Y$, et al. The association between continuity of care in the community and health outcomes: a population-based study. Isr J Health Policy Res 2012;1:21.

22. Jee SH, Cabana MD. Indices for continuity of care: a systematic review of the literature. Med Care Res Rev 2006;63:158-88.

23. Reid R, Haggerty J, McKendry R. Defusing the confusion: concepts and measures of continuity of healthcare: final report. Ottawa, ON: Canadian Health Services Research Foundation, 2002. http://www.cfhi-fcass.ca/Migrated/PDF/ResearchReports/ CommissionedResearch/cr contcare e.pdf

24. Salisbury C, Sampson F, Ridd M, et al. How should continuity of care in primary health care be assessed? Br J Gen Pract 2009;59: e134-41.

25. Imbens GW, Lemieux T. Regression discontinuity designs: a guide to practice. J Econom 2008;142:615-35.

26. Dong Y. Regression discontinuity applications with rounding errors in the running variable. J Appl Econom 2015;30:422-46.

27. Gelman A, Imbens G. Why high-order polynomials should not be used in regression discontinuity designs. NBER working paper series, no. 20405. Cambridge (MA): National Bureau of Economic Research, 2014.

28. Bland JM, Altman DG. Statistics notes: bootstrap resampling methods. BMJ 2015;350:h2622.
29. NHS Employers. 2014/15 General Medical Services (GMS) Contract Quality and Outcomes Framework (QOF). Published Online First: 2014. http://digital.nhs.uk/media/14019/QOF-Guidance-GMSContract-2014-15/pdf/QOF guidance_GMS contract_2014_15.pdf

30. Bardsley M, Steventon A, Doll H. Impact of telehealth on general practice contacts : findings from the whole systems demonstrator cluster randomised trial. BMC Health Serv Res 2013;13:1

31. Vandenbroucke JP, le Cessie S. Regression discontinuity design: Let's give it a try to evaluate medical and public health interventions. Epidemiology 2014;25:738-41.

32. Bloom HS. Modern regression discontinuity analysis. J Res Educ Eff 2012:5:43-82.

33. Ferris TG, Weil E, Meyer GS, et al. Cost savings from managing high-risk patients. In: Yong PL, Sanders RS, Olsen LA. The healthcare imperative: lowering costs and improving outcomes: workshop series summary. Washington (DC): The National Academies Press, 2010:301-10.

34. Goldman DP, Cutler D, Rowe JW, et al. Primary care physicians in ten countries report challenges caring for patients with complex health needs. Health Aff (Millwood) 2013;34:2104-12.

35. Martin S, Davies E, Gershlick B. Under Pressure. The Health Foundation. 2016. http://www.health.org.uk/publication/underpressure

36. Fincher RM, Lewis LA, Rogers LQ. Classification model that predicts medical students' choices of primary care or non-primary care specialties. Acad Med 1992;67:324-7.

37. Kassler WJ, Wartman SA, Silliman RA. Why medical students choose primary care careers. Acad Med 1991;66:41-3. http://www. scopus.com/inward/record.url?eid=2-s2.0-0026065158\&partnerID= 40\&md5=40994f8badc82b1726b1541d4657911e

38. Dias S, Sutton AJ, Welton NJ, et al. Evidence synthesis for decision making: heterogeneity--subgroups, meta-regression, bias, and bias-adjustment. Med Decis Making 2013;33:618-40.

39. Majeed A, Bardsley M, Morgan D, et al. Cross sectional study of primary care groups in London: association of measures of socioeconomic and health status with hospital admission rates. BMJ 2000;321:1057-60. 